\title{
Daily Weather Forecasting using Artificial Neural Network
}

\author{
Meera Narvekar ${ }^{1}$, Priyanca Fargose ${ }^{2}$ \\ Assistant Professor ${ }^{1}$, M.E. (CS) student ${ }^{2}$ \\ D.J. Sanghvi college of Engineering \\ Vile Parle, Mumbai-400056, Maharashtra, India
}

\begin{abstract}
Daily Weather forecasting is used for multiple reasons in multiple areas like agriculture, energy supply, transportations, etc. Accuracy of weather conditions shown in forecast reports is very necessary. In this paper, the review is conducted to investigate a better approach for forecasting which compares many techniques such as Artificial Neural Network, Ensemble Neural Network, Backpropagation Network, Radial Basis Function Network, General Regression Neural Network, Genetic Algorithm, Multilayer Perceptron, Fuzzy clustering, etc. which are used for different types of forecasting. Among which neural network with the backpropagation algorithm performs prediction with minimal error. Neural network is a complex network which is self-adaptive in nature. It learns by itself using the training data and generates some intelligent patterns which are useful for forecasting the weather. This paper reviews various techniques and focuses mainly on neural network with back propagation technique for daily weather forecasting. The technique uses 28 input parameters to forecast the daily weather in terms of temperature, rainfall, humidity, cloud condition, and weather of the day.
\end{abstract}

\section{Keywords}

Neural Network, Backpropagation Algorithm, Daily Weather Forecasting, ANN, Weather Prediction, Multilayer Neural Network, Quantitative Forecast.

\section{INTRODUCTION}

Weather forecasting is a process of identifying and predicting to a certain accuracy the climatic conditions using multiple technologies. Many of the live systems rely on weather conditions to make necessary adjustments in their systems. Forecasting helps to take necessary measures to prevent damage to life and property to a large extent. Quantitative forecast like temperature, humidity and rainfall are important in agriculture area, as well as to traders within commodity markets. Temperature forecasts are used by utility companies to estimate demand over coming days. Since outdoor activities are severely restricted by heavy rain, snow and the chill; forecasts can be used to plan activities around these events, and to plan ahead and survive them [11].

Nowadays multiple computing techniques are available which can be used for forecasting enhancing its accuracy. Different categories of forecasting methods are Naive approach, Judgmental methods, Quantitative and Qualitative method, Causal or econometric forecasting methods, Time series methods, Artificial intelligence methods, etc.

The weather forecast reports needs some intelligent computing which can read the nonlinear data and generate some rules and patterns to study and train from the observed data to predict the weather in future. Use of ANN will give results which are more accurate. Here, the error may or may not reduce completely. But, the accuracy will improve as compared to previous forecasts.

The weather forecasting is live forecasting where output of the model may be required for daily weather guide or weekly or monthly weather plans. Thus, the accuracy of the result is a very important aspect in this forecasting. Multiple issues are discussed which can be considered to get the accurate results. In Section two, a reviews multiple literature on weather forecasting. Section three introduces different terms about the neural network. Section four proposes a neural network model with all the specifications for forecasting weather with a high degree of accuracy.

\section{LITERATURE SURVEY}

This section reviews literature on different weather forecasting techniques using neural network.

Ch. Jyosthna Devi et al., 2012 presented an ANN based algorithm for predicting the temperature [1]. The BPN is used because it can fairly approximate a large class of functions. Authors propose a model which takes real time dataset with fifteen parameters as input, which is then normalized using min-max normalization to scale data between zeros to one. Then it is trained and tested using the Backpropagation Neural Network. The results are compared with the meteorological department to check the least error and accuracy of the model. It is found that the model has the potential for temperature forecasting.

Anomaly in this paper is that, it does not predict the complete weather condition like rainfall, clouds, etc.

Rainfall forecasting was done using Ensemble neural network (ENN) by Harshani R. K. Nagahamulla et al., 2012 [2]. In ENN, finite numbers of ANN are trained for the same task and their results are combined using the weighted average method. Here, each ANN is assigned a weight to minimize mean square error. The study area selected to be Colombo, where daily observed data of forty one years was used by dividing it in four climatic seasons every year with twenty six variables. The performance is compared with Backpropagation neural network (BPN), radial basis function network (RBFN) and general regression neural network GRNN). Results show that, ENN model predicts rainfall more accurately than individual BPN, RBFN and GRNN.

The paper compares ANN with BPN, RBFN and GRNN and the comparison shows that ANN gives more accuracy compared to others. 
A research was conducted by M. Nasseri et al., 2008 [3], in which they developed Feed forward type network to forecast rainfall using the backpropagation algorithm coupled with the Genetic Algorithm (GA). The study area selected was Sydney, Australia consisting fourteen recording rain gauges of four years data. Measurements of rainfall were at intervals of five minutes. After preliminary data analysis, twenty six storm events were selected for synchronization. Among which eighteen events were selected for training and four for testing. This paper concludes that multilayer perceptron (MLP) type network coupled with GA performs better than MLP type network alone.

It compares the performance of Multilayer Perceptron type networks and itself combined with GA. Thus, found that adding GA to MLP improves performance.

R Lee et al., 2004 [4] proposed an innovative, intelligent, multi-agent based environment named as intelligent Java Agent Development Environment (iJADE). It is used for weather prediction of eleven weather stations in Hong Kong using five years data which provides more than 7300 data records. The model uses GA for input node selection, a fuzzy classification for rainfall parameters and neural network for training using a BPN. Its experimental results are more promising than single point sources using similar network and other networks like Radial Basis Function Network, Learning Vector Quantization and Naive Bayesian Network. Authors compare ANN with RBF, LVQ and Naive Bayesian Network and prove that ANN results are better as compared to others.

Mohsen hayati et al., 2007 [5] uses ANN for one day ahead prediction of temperature. Here multilayer perceptron (MLP) is trained using $65 \%$ of patterns and tested using $35 \%$ of patterns for ten years meteorological data from Iran which was split into four seasons namely spring, summer, fall and winter. MLP network of three layers with sigmoid transfer function for hidden layers and linear transfer function for output layer is used. Number of hidden neurons and epochs were decided using the trial and error method. This paper concludes that MLP with this structure has minimum prediction error, good performance and reasonable prediction accuracy. The above paper uses historical data for ten years and achieves minimum error and reasonable accuracy.

Kumar Abhishek et al., 2012 [6] develops an ANN model to forecast average monthly rainfall. He selected data from Udupi, Karnataka which is eight months data for fifty years making 400 entries for input and output. The data is normalized by finding mean and standard deviation of each parameter. Then training is done on $70 \%$ of data and the remaining 30\% data is used for testing and validation. The model used is a three layered ANN with backpropagation learning. Later after testing, the results are compared with actual output. It showed higher degree of similarity in output. Thus it is proved that ANN model is accurate in prediction. Authors concluded that, learngdm is the best learning function for training whereas trainlm is the best training function.

The data used for research is huge enough i.e. of fifty years, thus, lager input data reduced the mean square error.

Yamin Wang et al., 2013 [7] proposes a novel wind speed forecasting method based on ensemble empirical mode decomposition (EEMD) and Genetic algorithm backpropagation Neural network.

The wind speed data recorded after every ten minutes is taken for five days which gives a total of 721 data for testing. The wind speed data is first decomposed by EEMD in eight different IMF's and a residue. Later GA $-\mathrm{BPNN}$ is used for forecasting each IMF. Then IMF results are combined and then the forecasting result for wind speed is obtained. Thus, this method proved to forecast wind speed more precisely as compared to traditional GA-BP and hybrid of EMD and GABP.

But here the forecasting is done considering only wind speed, which is not enough for weather forecasting.

Saima H. et al., 2011[8] reviews many hybrid methods used for weather forecasting with their merits and demerits. They used consortiums of statistical and artificial intelligent methods for achieving accuracy. Different models studied are

1. Hybrid autoregressive moving average (ARMA) model and ANN for wind speed prediction

2. Adaptive Neuro-Fuzzy Inference system (ANFIS) which is integration of Fuzzy Inference System and Neural network used for rainfall runoff prediction

3. Fuzzy clustering and Type-2 Fuzzy Logic

4. A Neuro evaluative Interval Type -2 TSK Fuzzy System

5. Grey Relational Analysis.

In all forecasting models, the major concern is with accuracy not the processing time. It is observed that not a single forecasting model can forecast with $100 \%$ accuracy, but they can reduce the accuracy error by various techniques.

Authors have compared all the techniques with their accuracy and found that no model can be totally accurate but near optimum results are expected.

Tony Hall et al., 1999 [9] develops ANN for Probability of Precipitation $(\mathrm{PoP})$ and Quantitative precipitation forecast (QPF) for Dallas-fort worth, Texas area. Neural Network was developed and initially two years data was used consisting nineteen variables. Later for three years the verification reports were generated. In this application, two networks are created, a QPF network for predicting amount of precipitation and a PoP network for probability or confidence in the forecast. The networks were designed with three features, first is a separate network for different seasons like warm and cool, and second is the use of QPF and PoP and the last is the network made interactive so we can rerun the entire network with some changes. This technique improves precipitation forecast dramatically, particularly for applications requiring accurate results.

Authors state that QPF and PoP together can improve the performance.

\section{ARTIFICIAL NEURAL NETWORK FOR WEATHER FORECASTING}

In Forecasting it is intuitive that accuracy is very important .The input parameters for a weather forecasting model are different Different types of data need different types of methods; and need to be handled accordingly. Statistical methods are usually associated with linear data whereas Artificial Intelligence methods are associated with nonlinear data [13]. Different learning models based on Artificial Intelligence are genetic algorithms, neuro-fuzzy logic and neural networks. Among which neural networks is preferred for time series forecasting for applications such as "stock index forecasting" in financial markets or "fault detection" in machine maintenance [14]. Weather forecasting can be done 
more accurately using ANN. Because daily weather data has multiple parameters representing temperature, humidity, rainfall amount, cloud distance and size, wind speed and direction, etc. All these parameters are not linear, but they need to be processed together to determine temperature, rainfall, humidity or weather status for the next day. Such type of applications need the models which are complex in nature and can produce the required result by generating the patterns on its own by performing self-learning using the training data given to the model.

To develop an ANN model for weather forecasting, selection of region for input data and parameters is necessary. The input data is to be taken from a specific area on which the model is trained and tested so that the model is able to generate accurate results. The number of input data given to model also helps to improve accuracy of the model by giving the results with a high degree of similarity between predicted and actual output data. The available data may be noisy thus, data should be cleaned. Similarly, it has to be normalized because, all the parameters are of different units and normalization will help the input and output parameters to correlate with each other [6]. The data should be divided in training and testing samples in proper proportion so that the results can be predicted, tested and validated properly. Structure of the NN model also has a great impact on generation of accurate results. The multilayer ANN helps in predicting nonlinear data more efficiently. The activation function will be different for different layers of $\mathrm{NN}$ as per need.

\section{ARTIFICIAL NEURAL NETWORKS (ANN)}

One type of network that sees the node as an 'artificial neurons' is called artificial neural networks. Artificial neural network is a software implementation which resembles the biological term central nervous system that is the human brain. Natural neurons receive signals through synapses located on the dendrites or membrane of the neuron. When the signals received are strong enough (surpass a certain threshold), the neuron is activated and emits a signal though the axon. This signal might be sent to another synapse, and might activate other neurons [15].

The complexity of real neurons is highly abstracted when modelling artificial neurons. These basically consist of inputs (like synapses), which are multiplied by weights (strength of the respective signals), and then computed by a mathematical function which determines the activation of the neuron.

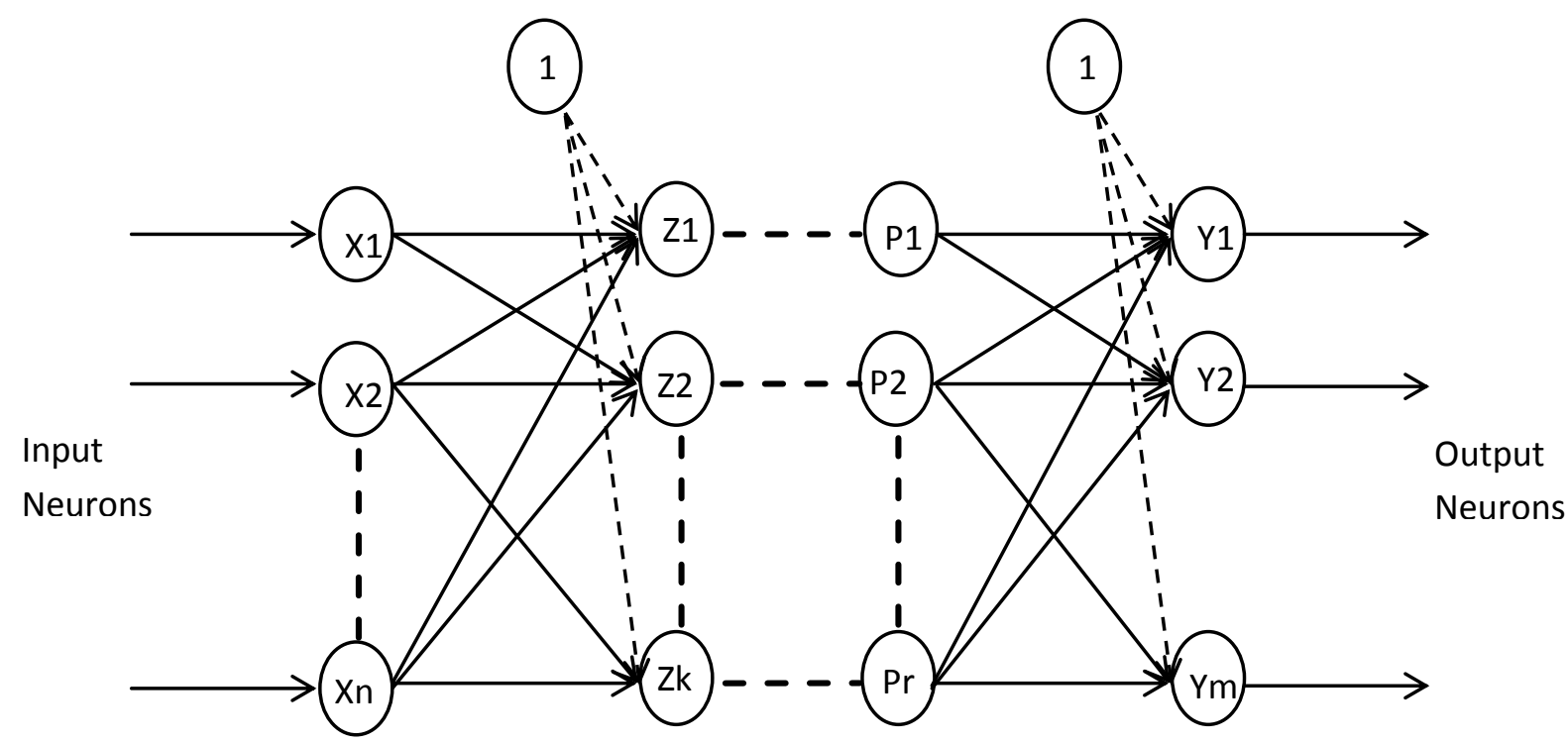

Figure 1: Neural Network structure

Another function computes the output of the artificial neuron (sometimes in dependence of a certain threshold).

This network is of a very complex type because it contains multiple neurons associated with each other in a well-formed structure to produce complex output with minimal error.

This network is mostly used to make predictions by training the model using past dataset and experience. Above Figure 1 shows an ANN structure where multiple input parameters are used as input neurons, which are then multiplied by weights and forwarded to a hidden layer where activation function is applied and then forwarded to output layer with another activation function where it finally computes the output of artificial neuron.

\section{THE BACKPROPAGATION OF ERROR ALGORITHM PROPOSED APPROACH}

The backpropagation algorithm is used in layered feedforward ANNs. It uses supervised learning, which means the model trains itself with the use of target output. For every set of input data the target output is provided. The neural network model processes the input data with random values for weights and suitable activation function using one or more hidden layer in between and then produces the predicted output. This predicted output is then compared with the target output provided for same input dataset. Thus, error is calculated by subtracting predicted output from target output. Using this error, the weights are adjusted and again the entire process is repeated for multiple epochs until the error is minimal or in acceptable range [15]. 
The idea of the backpropagation algorithm is to reduce this error, until the ANN learns the training data. The training begins with random weights, and the goal is to adjust them so that the error will be minimal.

For practical reasons, ANNs implementing the backpropagation algorithm do not have too many layers, since the time for training the networks grows exponentially. Also, there are refinements to the backpropagation algorithm which allow a faster learning.

\section{BACKPROPAGATION APPROACH}

The model proposed in this paper for weather forecasting using ANN using BP algorithm is as given below in Figure 2. The area for input data can be any one of a meteorological station area in which all the data is limited to a certain region. The different input parameters are taken viz. temperature, relative humidity, air pressure, wind speed and direction, cloud amount and height, rainfall, etc.

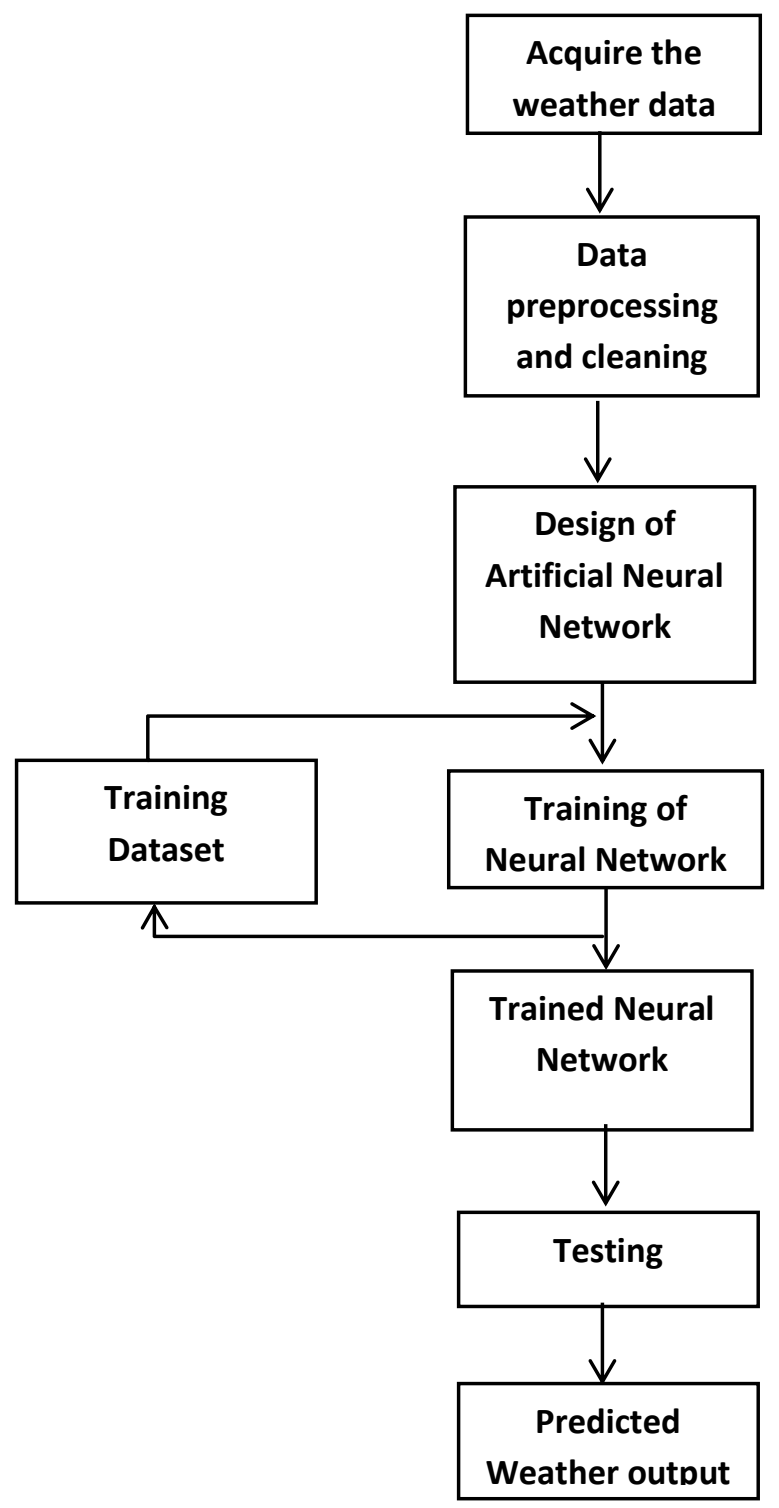

Figure 2: The Proposed Model for weather forecasting using Neural Network.

Input data is then pre-processed and cleaned. That means it is checked with any outlier and that is removed, missing values are entered, and data is checked if it is in the given range for the given parameter. Later ANN is designed with number of input and output nodes, hidden layers, activation function, and maximum number of epochs, weights, bias, goal and learning function. Neural network is trained with seventy percentages of the input data. Where the model is trained using this observed data to forecast the weather, followed by testing done using remaining thirty percentages of input data. Then the mean squared error and accuracy is calculated for the model by comparing the output of testing with target output.

This model generates output in terms of minimum and maximum temperature of the day, relative humidity and rainfall. The intensity of rainfall is represented by ten different classes as shown in Table 1 and the sky condition in five classes representing cloud status as given in Table 2.The range of classes for intensity of rainfall and cloud status ranges are taken from the Indian meteorological department because the input dataset is also from same source.

Seventy percentages of the dataset will be used for training and the other thirty percentages of the dataset will be used for testing and validation. Hidden layers are required for processing nonlinear data. Number of hidden layers in a network should be selected on a trial and error basis. Because as we increase the number of hidden layers; complexity of the network increases.

Table 1. Intensity of Rainfall

\begin{tabular}{|c|c|}
\hline Descriptive Term used & Rainfall amount (mm) \\
\hline No Rain & 0.0 \\
\hline Very light Rain & $0.1-2.4$ \\
\hline Light Rain & $2.5-7.5$ \\
\hline Moderate Rain & $3.6-35.5$ \\
\hline Rather Heavy & $64.5-124.4$ \\
\hline Heavy Rain & $124.5-244.4$ \\
\hline Very Heavy Rain & $>244.5$ \\
\hline Extremely Heavy Rain & $\begin{array}{c}\text { When the amount is a value } \\
\text { near about the highest } \\
\text { recorded rainfall at or near } \\
\text { the station for the month or } \\
\text { season. However, this term } \\
\text { will be used only when the } \\
\text { actual rainfall amount } \\
\text { exceeds 12 cm. }\end{array}$ \\
\hline
\end{tabular}

Table 2. Sky Conditions

\begin{tabular}{|c|c|}
\hline Sky condition & Value (Octa) \\
\hline Clear sky & 0 Octa \\
\hline Mainly clear & $1-2$ Octa of sky covered \\
\hline Partly cloudy & $3-4$ Octa of sky covered \\
\hline Generally cloudy / Mainly cloudy & $5-7$ Octa of sky covered \\
\hline Cloudy & $>7$ Octa of sky covered \\
\hline
\end{tabular}

The selection of number of neurons in each layer also plays an important role in model development. Because, a lesser number of neurons may cause the problem of "Underfitting" whereas, a greater number of neurons may cause 
"Overfitting". Thus, the number of hidden layer neurons can be decided by following the rule-of-thumb [10].

1. The numbers of hidden layer neurons are $2 / 3$ (or $70 \%$ to $90 \%$ ) of the size of the input layer. If this is insufficient then a number of output layer neurons can be added later on.

2. The number of hidden layer neurons should be less than twice of the number of neurons in an input layer.

3. The size of the hidden layer neurons is between the input layer size and the output layer size.

Weights and bias values are initially taken randomly and then during the training period values are adjusted automatically by comparing the mean squared error with the goal value defined. Learning rate trains the network with a constant value provided. Better results can be achieved with high accuracy when learning rate is smaller but its performance is slower. Activation functions are applied on each neuron to get the output of neuron on a given input in the neural network. The sigmoid function is a special case of logistic function which has a sigmoid curve. The sigmoid transfer function can be used for hidden layers and for the output layer the linear transfer function can be used.

The correctness and accuracy of the model can be checked using the Mean Squared error (MSE) function. The MSE measures the average of the squares of errors that is, the difference between the actual output and the predicted output of the model. Lesser the MSE value of the model, more accurate the results are.

\section{CONCLUSION AND DISCUSSION}

In this paper, different methods for weather forecasting are reviewed. ANN with backpropogation is recommended for weather forecasting.

ANN with backpropagation uses an iterative process of training where, it repeatedly compares the observed output with targeted output and calculates the error. This error is used to readjust the values of weights and bias to get an even better output. Hence this method tries to minimize the error. Thus, Artificial Neural network with Backpropagation algorithm seems to be most appropriate method for forecasting weather accurately.

The weather Forecasting has a big challenge of predicting the accurate results which are used in many real time systems like electricity departments, airports, tourism centers, etc. The difficulty of this forecasting is the complex nature of parameters. Each parameter has a different set of ranges of values. This issue is addressed by ANN. It accepts all complex parameters as input and generates the intelligent patterns while training and it uses the same patterns to generate the forecasts.

The Artificial Neural Network model proposed in this paper indicates all the parameters for input and output, training and testing data set, number of hidden layers and neurons in each hidden layer, weight, bias, learning rate and activation function. The Mean Squared Error between predicted output and the actual output is used to check accuracy.

\section{REFERENCES}

[1] Ch.Jyosthna Devi, B.Syam Prasad Reddy, K.Vagdhan Kumar, B.Musala Reddy, N.RajaNayak, "ANN Approach for Weather Prediction using Back Propagation," International Journal of Engineering Trends and Technology- Volume3Issue1- 2012.
[2] Harshani R. K. Nagahamulla, Uditha R. Ratnayake, AsangaRatnaweera," An Ensemble of Artificial Neural Networks in Rainfall Forecasting," The International Conference on Advances in ICT for Emerging Regions ICTer 2012: 176-181

[3] M. Nasseri, K. Asghari, M.J. Abedini, "Optimized scenario for rainfall forecasting using genetic algorithm coupled with artificial neural network," Elsevier, ScienceDirect, Expert Systems with Applications 35 (2008) 1415-1421

[4] R Lee, J Liu, "iJADEWeatherMAN: A Weather Forecasting System Using Intelligent Multiagent-Based Fuzzy Neuro Network", IEEE 181 Transactions on Systems, Man and Cybernetics - Part C: Applications and Reviews, vol 34, no 3, 369 - 377, August 2004.

[5] Mohsen hayati and Zahra mohebi, "Temperature Forecasting based on Neural Network Approach”, World Applied Sciences Journal 2(6): 613-620, 2007, ISSN 1818-4952, IDOSI Publications, 2007.

[6] Kumar Abhishek, Abhay Kumar, Rajeev Ranjan, Sarthak Kumar, "A Rainfall Prediction Model using Artificial Neural Network", IEEE Control and System Graduate Research Colloquium (ICSGRC 2012), pp 82-87.

[7] Yamin Wang, Shouxiang Wang, Na Zhang, "A Novel Wind Speed Forecasting Method Based on Ensemble Empirical Mode Decomposition and GA-BP Neural Network", 978-1-4799-1303-9/13/@2013 IEEE.

[8] Saima H., J. Jaafar, S. Belhaouari, T.A. Jillani, "Intelligent Methods for Weather Forecasting: A Review”, 978-1-4577-1884-7/11/@2011 IEEE.

[9] Tony Hall, Harold E. Brooks, Charles A. Doswell, " Precipitation Forecasting Using a Neural Network", Weather and Forecasting, Volume 14, June 1999, pp 338-345.

[10] SaurabhKarsoliya, "Approximating Number of Hidden layer neurons in Multiple Hidden Layer BPNN Architecture", International Journal of Engineering Trends and Technology- Volume3Issue6- 2012, pp 714717.

[11] [online]http://en.wikipedia.org/wiki/Weather forecasting

[12] Hansoo Lee, Jungwon Yu, YeongsangJeong, Sungshin Kim, "Genetic based feed-forward neural network training for chaff cluster detection," International conference of fuzzy theory and applications, Taichung, Taiwan, Nov.16-18, 2012

[13] R. Sallehuddin, et al., "Forecasting Time Series data using Hybrid Grey Relational Artificail Neural Network and Auto Regressive Integrated Moving Average," Journal of Applied Artificial Intelligence, vol. 23.

[14] J. N. K. Liu and K. Y. Sin, "Fuzzy neural networks for machine maintenance in mass transit railway system," IEEE Trans. Neural Networks, vol. 8, pp. 932-941, July 1997.

[15] Carlos Gershenson, "Artificial Neural Network for Beginners”, C.Gershenson@sussex.ac.uk, university of sussex. 